\title{
Fuzzy Logic Controller Stability Analysis Using a Satisfiability Modulo Theories Approach
}

\author{
Timothy Arnett ${ }^{1}$ and Brandon Cook $^{2}$ \\ University of Cincinnati, Cincinnati, OH, 45220 \\ Matthew Clark ${ }^{3}$ \\ Air Force Research Laboratory, Wright-Patterson AFB, OH, 45433 \\ and \\ Kuldip Rattan ${ }^{4}$ \\ Wright State University, Dayton, OH, 45435
}

\begin{abstract}
While many widely accepted methods and techniques exist for validation and verification of traditional controllers, at this time no solutions have been accepted for Fuzzy Logic Controllers (FLCs). Due to the highly nonlinear nature of such systems, and the fact that developing a valid FLC does not require a mathematical model of the system, it is quite difficult to use conventional techniques to prove controller stability. Since safety-critical systems must be tested and verified to work as expected for all possible circumstances, the fact that FLC controllers cannot be tested to achieve such requirements poses limitations on the applications for such technology. Therefore, alternative methods for verification and validation of FLCs needs to be explored. In this study, a novel approach using formal verification methods to ensure the stability of a FLC is proposed. Main research challenges include specification of requirements for a complex system, conversion of a traditional FLC to a piecewise polynomial representation, and using a formal verification tool in a nonlinear solution space. Using the proposed architecture, the Fuzzy Logic Controller was found to always generate negative feedback, but inconclusive for Lyapunov stability.
\end{abstract}

\section{Nomenclature}

$=$ pendulum angle

$=$ pendulum angle rate

$=$ pendulum angle error

$=$ pendulum angle error rate

$=$ gravitational acceleration

$=$ pendulum length

$=$ pendulum mass

\section{Introduction}

$\mathrm{I}$ $\mathrm{n}$ recent years, complex control methods have developed more quickly than the methods used to analyze them to ensure adherence to safety and performance requirements. While many analytical methods have been used to prove that a classical controller can achieve stability or other performance measures, at this time there are no established analytical methods for the analysis of complex non-linear controllers, such as ones using Fuzzy Logic.

${ }^{1}$ Graduate Student, AEEM, 745 Baldwin Hall, Cincinnati, OH 45221, AIAA Student Member.

${ }^{2}$ Graduate Student, Aerospace Engineering and Engineering Mechanics, Univeristy of Cincinnati, and Research Aerospace Engineer, NASA, Brandon.Cook@nasa.gov, AIAA Student Member.

${ }^{3}$ Chief, Autonomous Control Branch (RQQA), 2210 Eighth St., WPAFB, OH 45433.

${ }^{4}$ Professor, Department of Electrical Engineering, Dayton, OH 45435 
Although several methods exist for building evidence for controller verification using simulation or linearization, this cannot guarantee that the controller will act as desired for all possible conditions. Therefore, in this study we aim to develop a low level method for showing that a non-linear controller, such as a Fuzy Logic Controller (FLC), can be verified, or definitively refuted, for two cases of stability analysis: negative feedback and Lyapunov stability.

Although copious amounts of literature exists that deals with Lyapunov stability of controllers, including nonlinear ones, the primary focus of this study is to address the possible solutions to proving stability in hybrid systems that are analogous to FLCs. In a paper by Clark et $\mathrm{al}^{3}$, the stability of a FLC that has been converted into a hybrid representation is analyzed using the Lyapunov indirect method. This is achieved by using simulation trace data in order to give actual values for the state variables throughout the simulation. These data are then fed into a model checker and a formalized sentence is constructed. Using this formalized sentence a P matrix can be found that proves that the system, or some linearized version of it, is stable in the sense of Lyapunov. The sentence is constructed in such a way that the desired behavior is negated, and if a condition exists that violates it (i.e. a P matrix that satisfies the constraints for stability), the model checker will find a counterexample. This counterexample is thus the P matrix that shows the system is stable in the sense of Lyapunov. The multiple modes of the Fuzzy controller are taken into account during simulation so the data used in model checking includes values from each mode. One possible shortcoming of this method is that it relies on the indirect method which applies to linearized systems.

As opposed to the indirect method proposed by Clark et $\mathrm{al}^{3}$, in this study a direct method was used with a prescribed nonlinear candidate function. A Satisfiability Modulo Theories (SMT) solver, Z3, was used to evaluate the candidate function to check if it met the constraints for stability. The benefit of using this type of solver is that it provides formal proofs that the constraints will be met, or not, over the entire solution space. The authors are currently working on using the direct method with a general form function to solve for parameters that will constitute a global Lyapunov function if it exists. This approach will be added into the final manuscript.

In the remainder of this section, a description of the dynamic system used in this study is given, as well as a brief introduction to positive feedback and Lyapunov Stability. In Section II, the problem formulation and proposed solution is introduced. Section III highlights the methods for testing the proposed solution. In Section IV, the results of the study and a discussion of significant findings are presented. Lastly, in Section V, conclusions are drawn and areas for future work are described.

\section{A. Dynamic System Description}

The dynamic system that was analyzed in this study was a single degree-offreedom pendulum. In this system, an input torque was supplied by a controller to keep the pendulum inverted throughout time. For this system, two controller types were developed and tested: a classical Proportional-Derivative (PD) controller and a non-linear Fuzzy Logic Controller (FLC). The configuration of the dynamic system setup can be see in Figure 1.

While this system seems to be fairly simple in nature, the non-linear controller that was developed imposed interesting characteristics not typically seen by a classical controller. Simulation runs showed that the performance of the non-linear controller was superior to the classical linear controller. In each of these cases, experiments were conducted to show that the controller is able to meet the system requirements set forth by the designer.

\section{B. Positive Feedback}

In a dynamic system, a controller is created to provide negative feedback to the system. In essence, this means that the controller is driving both the state error and error rate to zero at all times. Therefore, if for any case the controller produces positive feedback, this is undesirable and will force the system to diverge from its equilibrium, or desired, location. If positive feedback occurs, this does not necessarily mean that the system is unstable, for it could push the system into a limit cycle; however, this is not a desired output of the system as it is still necessarily worsening performance.

\section{Lyapunov Stability}

The second area chosen for stability analysis in this study was testing for Lyapunov stability. This testing criteria is achieved by selecting a candidate function that is positive semi-denite and often includes a notion of energy in the system. To check stability in the sense of Lyapunov, the time derivative of the candidate function is found. If this function can be proven to be negative-definite over all conditions of interest, the system will be asymptotically 
stable $^{1,2}$. Therefore, as time goes on the controller will force the system to converge to zero error and error rate. Similarly, if the function is negative semi-definite the system is stable in the sense of Lyapunov and will remain in a set region around the desired position. Lastly, if the function is found to be zero or positive for any conditions of interest, the stability of the system cannot be concluded using the candidate function chosen. However, there is no guarantee that such a function can be found, or even exists, that satisfies these conditions for any given system.

\section{Proposed Solution}

Prior to developing the controllers for the inverted pendulum system, a model for the single degree of freedom pendulum was developed using a Lagrangian approach. In this approach, energy methods are used to develop the equation of motion. This equation of motion was found to be:

$$
\frac{1}{3} m L^{2} \ddot{\theta}-m g \frac{L}{2} \sin \theta=F
$$

Where $g$ is the acceleration due to gravity and $F$ is the applied torque (i.e. the output from the controller). With this model, the two controllers were developed to keep the pendulum inverted: a Proportional-Derivative Controller (PD) and a Fuzzy Logic Controller (FLC).

\section{A. PD Controller Development}

The first that was created was the linear PD controller that has the form:

$$
F=K_{p} e_{\theta}+K_{d} \dot{e}_{\theta}
$$

Where $K_{p}$ and $K_{d}$ are real-valued gains specified by the designer and $F$ is the controller output. Using traditional tuning techniques, the gains were selected to be: $K_{p}=113, K_{d}=7.6$ to stabilize the system.

\section{B. Fuzzy Controller Development}

Next, a Mamdani-type Fuzzy Logic Controller, based on Fuzzy Set Theory introduced by Lofti Zadeh in the 1960's, was developed. A Fuzzy controller uses input classification and rules associating different fuzzy sets to produce complex and highly non-linear inputoutput relationships. Using a Fuzzy controller has many benefits including the ability to be developed by a human designer that has some knowledge about how the system should desirably act. However, the designer does not need to formulate an exact mathematical model that is necessary for classical controllers. Not having to develop a model of the system can be quite beneficial, especially in the cases where the physical system is complex.

While a Fuzzy system can be developed without exact

\begin{tabular}{|l|l|l|l|l|l|l|l|}
\hline \multicolumn{1}{|c|}{} & & & & & & & \\
\hline de/dt & NB & NM & NS & ZE & PS & PM & PB \\
\hline NB & NB & NB & NB & NM & NM & NS & ZE \\
\hline NM & NB & NB & NM & NM & NS & ZE & PS \\
\hline NS & NB & NM & NM & NS & ZE & PS & PM \\
\hline ZE & NM & NM & NS & ZE & PS & PM & PM \\
\hline PS & NM & NS & ZE & PS & PM & PM & PB \\
\hline PM & NS & ZE & PS & PM & PM & PB & PB \\
\hline PB & ZE & PS & PM & PM & PB & PB & PB \\
\hline
\end{tabular}

Figure 2. FLC rule base

knowledge on how the system dynamics work, the input-output relationship can be complicated; therefore, the relationship cannot be analyzed like a traditional controller. Due to this, we aim to develop an approach for analyzing these input-output relationships. This is necessary for these types of systems to ensure they perform as expected prior to being implemented into safety-critical systems.

In each fuzzy system, the user must define a rule base to govern the action of the controller for a set of sensory inputs. The Fuzzy system used in this study was trained using a combination of expert knowledge and Genetic Algorithms, both of which are beyond the scope of this paper.

The final rule base governing the FLC can be seen in Figure 2. Where "NB" is "negative big", "NM" is "negative medium", "NS" is "negative small", "ZE" is "zero", "PS" is "positive small", "PM" is "positive medium", and "PB" is "positive big".

\section{Conversion to Hybrid Representation}

In order to somewhat simplify the input-output relationship, the FLC was constrained and converted to a hybrid system using previously developed methods ${ }^{4}$. This conversion allows the FLC to be represented by a piecewise polynomial function. The constraints for the FLC are a particular fuzzy partitioning with symmetric triangular 
membership functions, normalized domains for the fuzzy membership sets, and uses the weighted average defuzzification method with product rule scaling of the output membership functions. Since fuzzy controllers have discrete transition

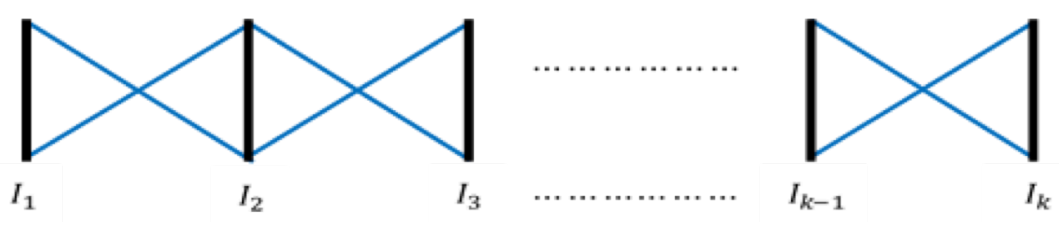

Figure 3. Input membership functions with fuzzy partitioning

points between different modes, and each mode contains a continuous function, they are essentially a hybrid system. By converting our FLC to a hybrid system, we now have the tools necessary to analyze each controller mode separately. An example of the fuzzy partitioned FLC can be seen in Figure 3.

This drastically simplifies the input-output relationships compared to typical FLC implementations and can be formalized using an SMT solver, such as Z3. The input-output relationship has the form:

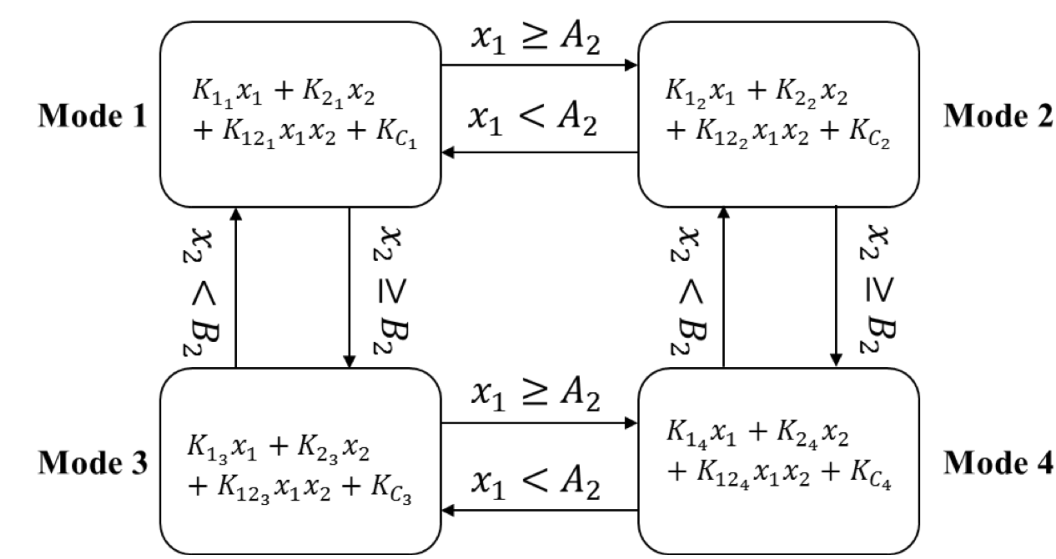

Figure 4. Hybrid representation of FLC with continuous modes and discrete transitions

$$
K_{1} x_{1}+K_{2} x_{2}+K_{12} x_{1} x_{2}+K_{c}
$$

Where each coefficient, $K$, is a constant for a particular mode. The modes are defined as the regions between the center points of the input membership functions. The transitions occur such that the functions that govern each mode are piecewise continuous and are inclusive on the lower boundary. The exception to this is the "upper" mode, when either input is in its "PB" membership function, where the domain is inclusive on both boundaries. A visualization of the hybrid system transitioning between these modes is shown in Figure 4.

However, we can see that the input-output relation is still non-linear, and will therefore require non-traditional stability analysis.

\section{E. Formalizing Constraints into First Order Logic}

Once the controllers were developed, we then formalized the system equations, and the constraints for each stability criteria, for both controllers into first order logic. Using these sentences, we now need to show that the equations always hold over the bounded domain. This can be done by negating the entire statement and then proving that the statement and its constraints are unsatisfiable. As previously mentioned in the introduction to positive feedback requirements, the controller shall always drive the error and error rate towards zero. This expression can be formalized using the following first order logic sentence:

$$
\forall e_{\theta} \forall \dot{e}_{\theta}\left(\begin{array}{l}
\left(\left(\operatorname{sgn}\left(e_{\theta}\right) \wedge \operatorname{sgn}\left(\dot{e}_{\theta}\right)\right) \rightarrow \operatorname{sgn}(F)\right) \wedge \ldots \\
\left(\left(\neg \operatorname{sgn}\left(e_{\theta}\right) \wedge \neg \operatorname{sgn}\left(\dot{e}_{\theta}\right)\right) \rightarrow \neg \operatorname{sgn}(F)\right)
\end{array}\right)
$$


Therefore, this can be verified by examining the controller input-output equation(s). This approach was tested for each controller type. For the case of checking that the system is stable in the sense of Lyapunov, a candidate function was created to meet the set criteria. Our candidate function can be seen as follows:

$$
V(\bar{x})=\frac{1}{2} x_{2}^{2}+\frac{1}{2}\left(x_{1}+x_{2}\right)^{2}+2\left(1-\cos x_{1}\right)
$$

This function meets the constraints for a candidate Lyapunov function that $V(0)=0$ and $V(x \neq 0)>0$. We can now find $\dot{V}(x)$ using the following expression:

$$
\dot{V}(\bar{x})=\frac{d V}{d \bar{x}} \overline{\dot{x}}
$$

Where $x_{1}=\theta$ and $x_{2}=\dot{\theta}$. With this candidate Lyapunov function, we now must verify that $\dot{V}(x)$ is strictly less than 0 . Thus, our constraint equation for testing stability in the sense of Lyapunov becomes:

$$
x_{2}\left(x_{1}+x_{2}+2 \sin x_{1}\right)+\left(x_{1}+2 x_{2}\right)\left(\frac{3}{m L^{2}}\left(F+\frac{m g L}{2} \sin x_{1}\right)\right)<0
$$

Lastly, after the constraint equations had been written in first order logic, the representations of each controlled system and constraints were implemented into a Satisfiability Modulo Theories (SMT) Solver - Z3 $3^{5}$ to verify that the system always meets the specification criteria.

\section{Testing}

\section{A. Positive Feedback}

To verify that the linear PD controller never had positive feedback for all possible inputs, a Z3 script was developed to verify our constraint satisfaction problem. Therefore, the inputs of error and error rate first had to be declared within the Z3 architecture. To do this, the input $x_{1}$ was used to represent $e_{\theta}$, and $x_{2}$ was used to represent $\dot{e}_{\theta}$. Once this was complete, the assertions that the output torque from the controller was always in the desired direction was created. That is, if both $x_{1}$ and $x_{2}$ were positive, the torque must always be positive. On the other hand, if both $x_{1}$ and $x_{2}$ were negative, the torque must necessarily be negative to ensure negative feedback. By setting the hard constraints on the ranges of $x_{1}$ and $x_{2}$ for each case (i.e. strictly negative or strictly positive), the constraints on the controller output could be negated, resulting in the following expression:

$$
\forall e_{\theta} \forall \dot{e}_{\theta} \neg\left(\begin{array}{l}
\left(\left(\operatorname{sgn}\left(e_{\theta}\right) \wedge \operatorname{sgn}\left(\dot{e}_{\theta}\right)\right) \rightarrow \operatorname{sgn}(F)\right) \wedge \ldots \\
\left(\left(\neg \operatorname{sgn}\left(e_{\theta}\right) \wedge \neg \operatorname{sgn}\left(\dot{e}_{\theta}\right)\right) \rightarrow \neg \operatorname{sgn}(F)\right)
\end{array}\right)
$$

Thus, if the SMT solver was used to check if the above system is satisfiable, and found it to be true, positive feedback can occur in the system. However, if it is found to be unsatisfiable, the system is guaranteed to have negative feedback for all inputs within the analyzed domain.

Similarly, the possibility of having positive feedback occurring in the Fuzzy controller case was analyzed using the same approach. The only difference here is that the Fuzzy has $\left(n_{1}-1\right)\left(n_{2}-1\right)$ possible modes that need to be analyzed, where $n_{1}$ and $n_{2}$ are the number of membership functions for each respective input; as opposed to the PD case that only had one mode.

\section{B. Lyapunov Stability}

Finally, both controllers were tested for Lyapunov stability. Using the equation previously defined for $\dot{V}(x)$ and constraining the inputs to the controller, we can develop a constraint satisfaction problem to ensure that $\dot{V}(x)$ is strictly negative. Here, we again negate the constraint satisfaction problem resulting in the following expression: 


\begin{tabular}{|c|c|c|c|c|c|c|c|}
\hline $\mathrm{de} / \mathrm{dt}$ & NB & NM & NS & ZE & PS & PM & PB \\
\hline NB & PB & NB & NB & NM & NM & NS & ZE \\
\hline NM & NB & NB & NM & NM & NS & ZE & PS \\
\hline NS & NB & NM & NM & NS & ZE & PS & PM \\
\hline ZE & NM & NM & NS & ZE & PS & PM & PM \\
\hline PS & NM & NS & ZE & PS & PM & PM & PB \\
\hline PM & NS & ZE & PS & PM & PM & PB & PB \\
\hline PB & ZE & PS & PM & PM & PB & PB & PB \\
\hline
\end{tabular}

$$
\begin{aligned}
\forall x_{1} \forall x_{2} \neg\left(x_{2}\left(x_{1}+x_{2}+2 \sin x_{1}\right)\right. \\
\left.\quad+\left(x_{1}+2 x_{2}\right)\left(\frac{3}{m L^{2}}\left(F+\frac{m g L}{2} \sin x_{1}\right)\right)<0\right)
\end{aligned}
$$

By proving that the above expression is unsatisfiable we can guarantee that the system is asymptotically stable. For this case, the controller output $\mathrm{F}$ was simply changed based on the type and mode of the controller. For the PD controller, only one mode exists, however, the Fuzzy controller used has 36 modes. These modes were checked along with the corresponding input bounds for unsatisfiability.

\section{Results}

\section{A. Positive Feedback}

Once each of the above cases was developed, they were analyzed using Z3. For the case where we were checking for positive feedback for the linear controller, Z3 found that in all cases the model was unsatisfiable. Therefore, we have proven for the domain ranges tested, we can ensure that the system will only produce negative feedback. When checking for positive feedback in the Fuzzy controller, we again found that the system was unsatisfiable in all modes analyzed. Thus, we again showed that the FLC would always produce negative feedback to the system.

As a counter example for the FLC positive feedback check, a bad rule was intentionally placed in the rule base, as seen in Figure 5.

By doing so, we would expect $\mathrm{Z3}$ to find that the constraint satisfaction problem indeed has a solution and is satisfiable. Upon doing so, we found that this was the case. This was used as a sanity check to ensure that we were formulating the problem correctly in Z3 and to check that Z3 could identify this potential hazard in the proposed Fuzzy controller.

\section{B. Lyapunov Stability}


Prior to evaluating both the PD and Fuzzy controllers using the Z3 formulation, a MATLAB simulation platform was developed to test a range of inputs for the system. In these cases, we wanted to ensure that the derivative of our candidate Lyapunov function was strictly negative for all points tested. Upon conducting this experiment, we found that both the PD and the Fuzzy controllers did in fact satisfy this condition for the points tested. A figure showing the results of the simulated candidate function for the controllers can be seen in Figure 6. Here, it can be seen that the candidate solution was strictly negative for all points evaluated in the simulation.

Next, the PD controller was tested for stability in the sense of the Lyapunov using Z3. When the program ran the same range of inputs that were analyzed in the simulation platform, we found that $\mathrm{Z3}$ found a condition that the simulation points missed. While the simulation platform analyzed a large number of input values, it does not analyze the entire range of inputs to infinite precision. Therefore, the simulation could easily miss points that make $\dot{V}(\bar{x})$ positive (i.e. violate the system requirement for stability).

Similarly, the Fuzzy implementation had the same outcome. In the simulation cases, the Fuzzy controller was strictly negative over the range of inputs, however, when realized and tested using first-order logic in Z3, two modes were found to be satisfiable. That is, the stability requirement was violated.

It is important to note that this does not mean that the controlled system is unstable; it simply means that no conclusions can be drawn about the system stability using our selected candidate Lyapunov function. If a candidate function could be found that does satisfy these requirements, we could then conclude that the system is stable (i.e. asymptotically or in the sense of Lyapunov). However, the power to identify these conditions that simulation results missed is also valuable when making claims about a system's adherence to its requirements.

\section{Conclusion}

Using the approaches described in this study, we have shown that $\mathrm{Z3}$ is a valid tool for analyzing several desirable (and undesirable) characteristics of a linear and non-linear controller. Z3 offers conclusive proofs that a
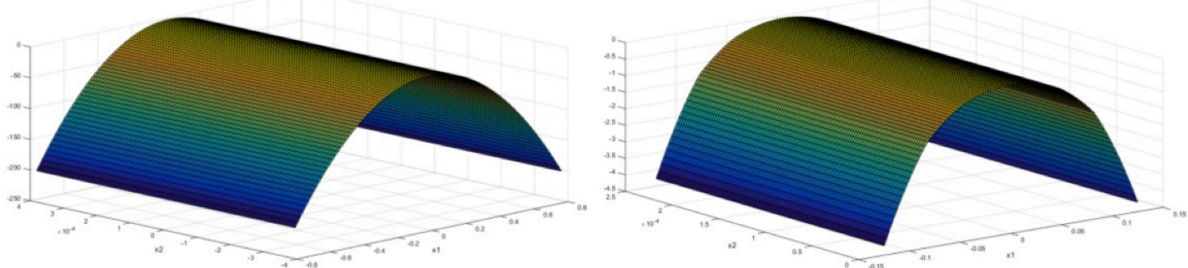

Figure 6. $\dot{\boldsymbol{V}}(\bar{x})$ for PD (left) and Fuzzy (right) controllers requirement holds or not, given that it can be properly represented in formal logic. We were able to show that both controllers do not give positive feedback by showing that conditions that do give positive feedback do not exist (i.e. they are unsatisfiable). Lastly, we were able to show that although simulation runs gave us strictly negative values for $\dot{V}(\bar{x})$, Z3 showed conditions exist where it is positive. Therefore, the controllers cannot be said to be asymptotically stable.

Although the results are inconclusive as far as proving Lyapunov stability given a prescribed candidate function, the authors are currently working on generating parameters for a general form candidate function that satisfies the constraint that its derivative is negative semi-definite or negative definite in all modes. This is similar to the work done with Lyapunov's indirect method mentioned previously, but the benefit with the approach presented in this study is that we can use a non-linear representation of the system (with the exception of the transcendental function). Our confidence level is very high that we will be able to do this and any results that are found will be included in the final manuscript.

\section{Acknowledgments}

T. Arnett thanks Matthew Clark, Dr. Kuldip Rattan, and Jon Hoffmann of AFRL for their help and previous work in the area. T. Arnett and B. Cook also thanks Dr. Kelly Cohen for his invaluable advice and support. This work was partially supported by the Dayton Area Graduate Studies Institute.

\section{References}

${ }^{1}$ Dahleh, M., "Lyapunov Methods," Dynamic Systems Recitation 6, Department of Electrical Engineering and Computer Science, Massachusetts Institute of Technology, Cambridge, MA, 2003 (unpublished).

${ }^{2}$ Seyfried, Aaron W. "Stability of a Fuzzy Logic Based Piecewise Linear Hybrid System", Thesis, Wright State University, 2013.

${ }^{3}$ K. S. Rattan, M. A. Clark and J. A. Hoffman, "Design and analysis of a multistage fuzzy PID controller," American Control Conference (ACC), 2015, Chicago, IL, 2015, pp. 5726-5731. 
${ }^{4}$ Clark, M.A. and Rattan, K.S., "Piecewise Affine Hybrid Automata Representation of a Multistage Fuzzy PID Controller", 2014 AAAI Spring Symposium Series, Palo Alto, CA, 2014.

${ }^{5}$ Z3, Software Package, Ver. 4.3.2, Microsoft, Redmond, WA, 2014. 